\title{
The Role of Serratus Anterior Plane Block During in Video-Assisted Thoracoscopic Surgery
}

\author{
Jia-qi Chen $\cdot$ Xin-lu Yang $\cdot$ Hai Gu $\cdot$ Xiao-qing Chai $\cdot$ Di Wang
}

Received: July 24, 2021 / Accepted: September 8, 2021 / Published online: September 19, 2021

(C) The Author(s) 2021

\section{ABSTRACT}

Although thoracoscopy has characteristics such as a small surgical incision and low stress response, post-surgical pain after a thoracoscopic operation is no less than that after a thoracotomy. Moreover, poor post-surgical pain management is likely to cause an increased incidence of postoperative pulmonary complications (PPCs) and chronic post-surgical pain. The serratus anterior plane block (SAPB) is a regional anesthesia method whereby local anesthetics (LAs) are injected into the serratus anterior space to block the lateral cutaneous branch of the intercostal nerve, long thoracic nerve, and dorsal thoracic nerve. The block range of the SAPB covers the incisions of videoassisted thoracoscopic surgery (VATS) and the site of the chest tube, which are often located in the antero-lateral chest wall. Therefore, the SAPB can achieve effective analgesia in VATS. For example, $0.125 \%$ to $0.25 \%$ levobupivacaine (20-25 ml) is widely used for thoracic surgery,

Jia-qi Chen, and Xin-lu Yang made equal contributions to the article.

J. Chen · X. Yang $\cdot$ H. Gu $\cdot$ X. Chai $\cdot$ D. Wang $(\bowtie)$

Pain Clinic, Department of Anesthesiology, First

Affiliated Hospital of USTC (Anhui Provincial

Hospital), University of Science and Technology of

China, 17 Lujiang Road, Luyang District, Hefei

230001, Anhui, China

e-mail: di.wang@ustc.edu.cn which can achieve effective analgesia and avoid adverse reactions. Moreover, it has advantages compared with thoracic segmental epidural block (TEA) and thoracic paravertebral block (TPVB), such as simple operation, increased safety, fewer complications, and hemodynamic stability. In addition, adequate analgesia is helpful for pulmonary function recovery and reduces the incidence of PPCs. This article introduces the anatomical mechanism of the SAPB, diverse operation approaches, how to choose drugs and adjuvants, and the resulting impacted area range. It summarizes the advantages and disadvantages of the SAPB compared with other analgesic methods and posits that the SAPB is beneficial to the recovery of postoperative lung function, which provides more options for postoperative analgesia after VATS.

Keywords: Serratus anterior plane block; Video-assisted thoracoscopic surgery; Postoperative acute pain management; Recovery of pulmonary function 


\section{Key Summary Points}

Acute pain after thoracic surgery is believed to negatively influence breathing and lung function in the early postoperative period. Therefore, acute pain after thoracic surgery should be prevented or reduced by better pain management, allowing patients to perform effective breathing exercises for adequate gas exchange postoperatively

Continuous SAPB, in which a catheter is placed and connected to a patientcontrolled analgesia device, provided a comparable analgesia to continuous thoracic epidural analgesia or patientcontrolled intravenous analgesia

In this narrative review, we first reviewed the characteristics of SAPB technically, such as anatomical mechanism, diverse operation approaches, how to choose drugs and adjuvants, and resulting impacted area range

In the context of perioperative period of video-assisted thoracoscopic surgery in particular, we further summarized the evidence on SAPB with acute post-surgical pain management and proposed that SAPB benefits postoperative lung function recovery by removing the pain limitation from ventilatory function practice

\section{INTRODUCTION}

Video-assisted thoracoscopic surgery (VATS) has been employed for thoracic surgery for several decades. It is now widely accepted owing to its small surgical incision, low stress response, and high tolerability [1]. Initially, chest pain elicited by VATS was thought to be reduced by the reduced inflammatory response and tissue damage of the thoracoscopic operation [2]. Unfortunately, most available evidence does not support the claim that the analgesic requirements of VATS are fewer than the measures required after an open thoracotomy [3, 4]. Similar to the situation in the open procedure, nociceptive stimuli during VATS also stem from multiple mechanisms owing to the chest incision, surgical trauma, and placement of the chest tube. Thus, the pathophysiology of acute pain after VATS should not differ much from that following open thoracotomy, and the resulting pain intensity is still thought to be moderate to severe, especially after major surgical procedures, such as lobectomies [5].

In the immediate postoperative period of VATS, poorly controlled post-surgical pain may lead to chronic post-surgical pain over time, and it also has a significant negative impact on respiratory mechanics, which potentially contributes to an increased risk of postoperative pulmonary complications (PPCs) [6]. A key aim for the enhanced recovery after surgery (ERAS) strategy of VATS is accelerating the recovery from functional respiratory impairment. There has been a greater appreciation for the relationship between pulmonary performance and movement-evoked pain in the immediate postsurgical period [7-9]. For example, pulmonary function exercise is encouraged but underestimated because it can reliably facilitate improvement in postoperative respiratory impairment. It has been commonly found that pulmonary function exercise is largely restricted once uncontrolled pain starts postoperatively, thereby leading to PPCs [10]. Conversely, early and timely pain control can ensure enforced breathing or free coughing, which is beneficial for improving lung atelectasis and accelerating the recovery of respiratory function [11].

Systemically administered opioids are the most commonly used drugs to reduce the postsurgical pain after VATS; however, they induce a series of adverse reactions, including postoperative nausea/vomiting and respiratory depression $[12,13]$. Considering the comorbidities in most patients eligible for thoracic surgery, it is critically important to lessen their use [14]. Consequently, a multimodal perioperative analgesia, administered regionally and systemically, has become a standard to mitigate postoperative pain [2]. In 2013, Blanco et al. [15] 
first proposed a novel regional block for anterolateral chest wall analgesia by injecting local anesthetics (LAs) into the surface of the anterior serratus muscle or deep interstitial space. This method, termed the serratus anterior plane block (SAPB), is promising for chest wall analgesia as it is technically easy to perform and can fully cover the surgical area impacted by VATS [16].

This narrative review first examines the technical characteristics of SAPB, such as the anatomical mechanism, diverse operational approaches, how to choose drugs and adjuvants, and the resulting impacted area range. Particularly, in the context of the VATS perioperative period, this evidence is further summarized on the SAPB with acute post-surgical pain management, and we offer the perspective that SAPB has a benefit on postoperative lung function recovery by reducing the pain caused by ventilatory function practice (Table 1 ).

A comprehensive literature review was performed in MEDLINE, PubMed, and Cochrane databases from 1981 to 2021 using the terms "serratus anterior plane block," "video-assisted thoracoscopic surgery," "thoracic surgery," "anatomy," "postoperative pulmonary complications," "continuous serratus anterior plane block," "regional anesthesia," "local anesthetic," "adjuvant," "approach," "ultrasound-guided," "thoracic segmental epidural block," "thoracic paravertebral block," and "post-surgical pain." All retrieved articles were reviewed by title, abstract, and the article itself when its content was not clearly indicated by the title and abstract. This article is based on previously conducted studies and does not contain any studies with human participants or animals performed by any of the authors.

\section{ANATOMICAL BASIS}

Serratus anterior is a quadrilateral broad flat muscle located on the lateral side of the chest wall. It runs from the lateral edge of the first to ninth ribs and ends at the medial edge of the ipsilateral scapula [17]. The upper part of the serratus anterior is covered by the pectoralis major and pectoralis minor muscles, and the inner part is adjacent to the intercostal muscles (Fig. 1) [18]. In addition, the thoracic spinal nerve roots penetrate from the intervertebral foramen, and they are subdivided into the ventral and dorsal branches. The dorsal branch innervates the skin and muscles in the

Table 1 Key recommendations for performing an SAPB

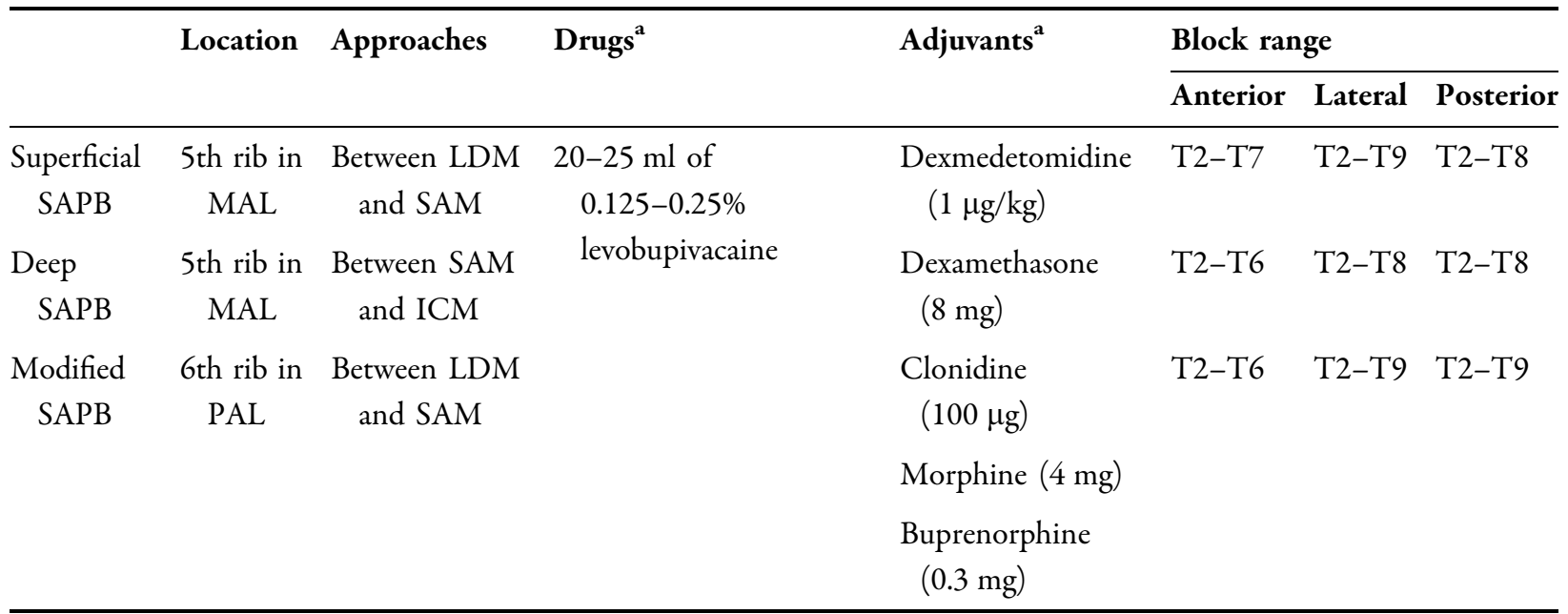

$M A L$ mid-axillary line, $P A L$ posterior axillary line, $L D M$ latissimus dorsi, $S A M$ serratus anterior muscle, $I C M$ intercostal muscle

a The recommended doses of drugs and adjuvant are approximate amount of range 


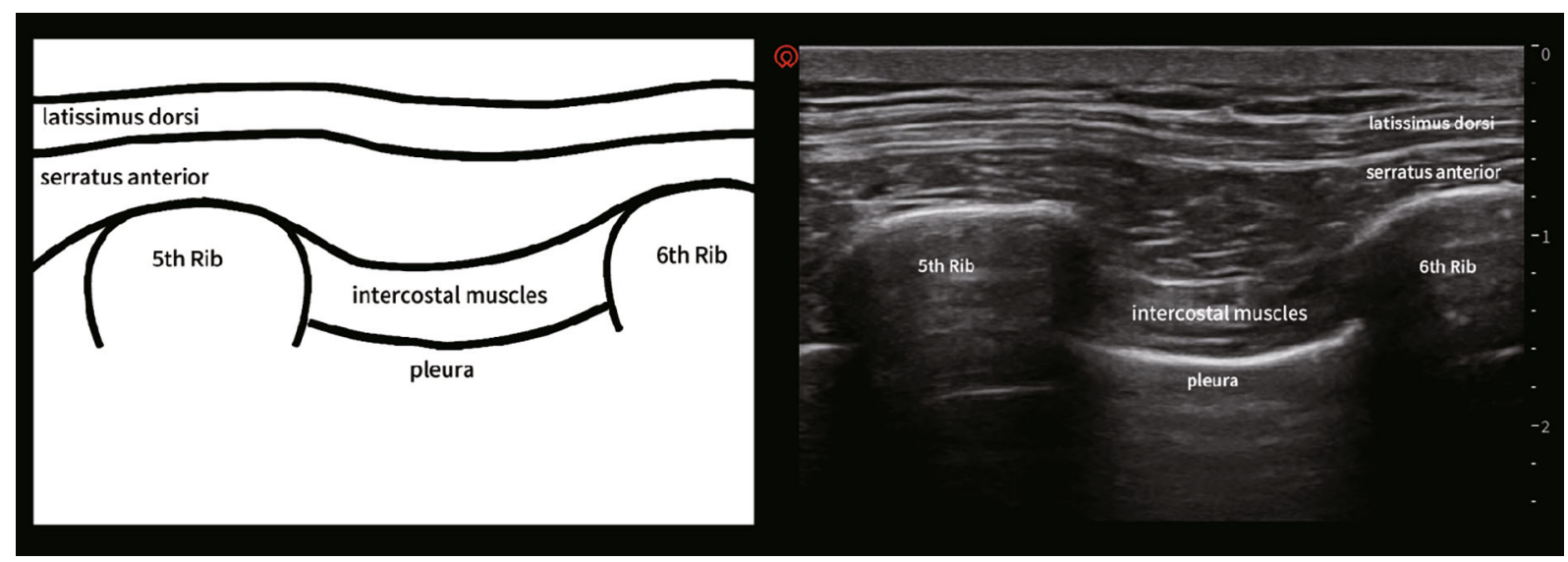

Fig. 1 Anatomical planes (left) and the ultrasound imaging (right) of SAPB. Cited from: Gao et al. [31]

paravertebral region, and the ventral branch continues to course with the intercostal nerve laterally. In the mid-axillary line, the intercostal nerve is subdivided into the lateral cutaneous branch, which passes through the intercostal muscle, and the serratus anterior muscle, which innervates the skin and muscles in the lateral chest wall [19]. In addition, the long thoracic nerve courses on the surface of the serratus anterior muscle, which runs along with the lateral thoracic artery and innervates the serratus anterior muscle. The dorsal thoracic nerve also courses on the surface of the serratus anterior muscle in the mid-axillary line [20]. Mayes et al. [21, 22] used cadaveric specimens to perform a diffusion test of a melanic solution in the serratus anterior interval showing the diffusion area, including the lateral cutaneous branches of the second to sixth intercostal nerves, the long thoracic nerve, and the dorsal thoracic nerve in the serratus anterior interval. Therefore, anatomically, the SAPB can block not only the lateral cutaneous branch of the intercostal nerve but also the long thoracic nerve and the thoracic dorsal nerve. This probably explains why SAPB can successfully cover the antero-lateral chest wall in most cases, which may not be usually achieved by other regional anesthesia methods, such as thoracic segmental epidural block (TEA) and thoracic paravertebral block (TPVB).

\section{ULTRASOUND-GUIDED BLOCK}

In recent years, image-guided procedures have emerged and played an active role in pain management. In particular, analgesia without systemic side effects can be achieved regionally by precise administration of LAs into the local area by ultrasound-guided techniques. These offer safe and effective methods for pain relief, allowing clinicians to control or reduce the amount of opioids used. In terms of techniques and new devices or technological improvements, new local regional options, such as SAPB, have emerged (Fig. 2).

\section{SAPB Approaches}

\section{Superficial SAPB}

The superficial serratus plane block was first proposed by Blanco et al. [15] in 2013. With the patient in a supine position, an ultrasound probe was placed at the level of the midclavicular line in a sagittal plane. The second rib was recognized at the axillary artery. The probe was moved downward to count the ribs until the level of the fifth rib in the mid-axillary line. At this time, the latissimus dorsi muscle and the serratus anterior muscle were clearly identified under ultrasound. Next, a 22-G needle was inserted between the latissimus dorsi and the serratus anterior muscles. Furthermore, 0.125\% levobupivacaine was injected at $0.4 \mathrm{ml} / \mathrm{kg}$, and 


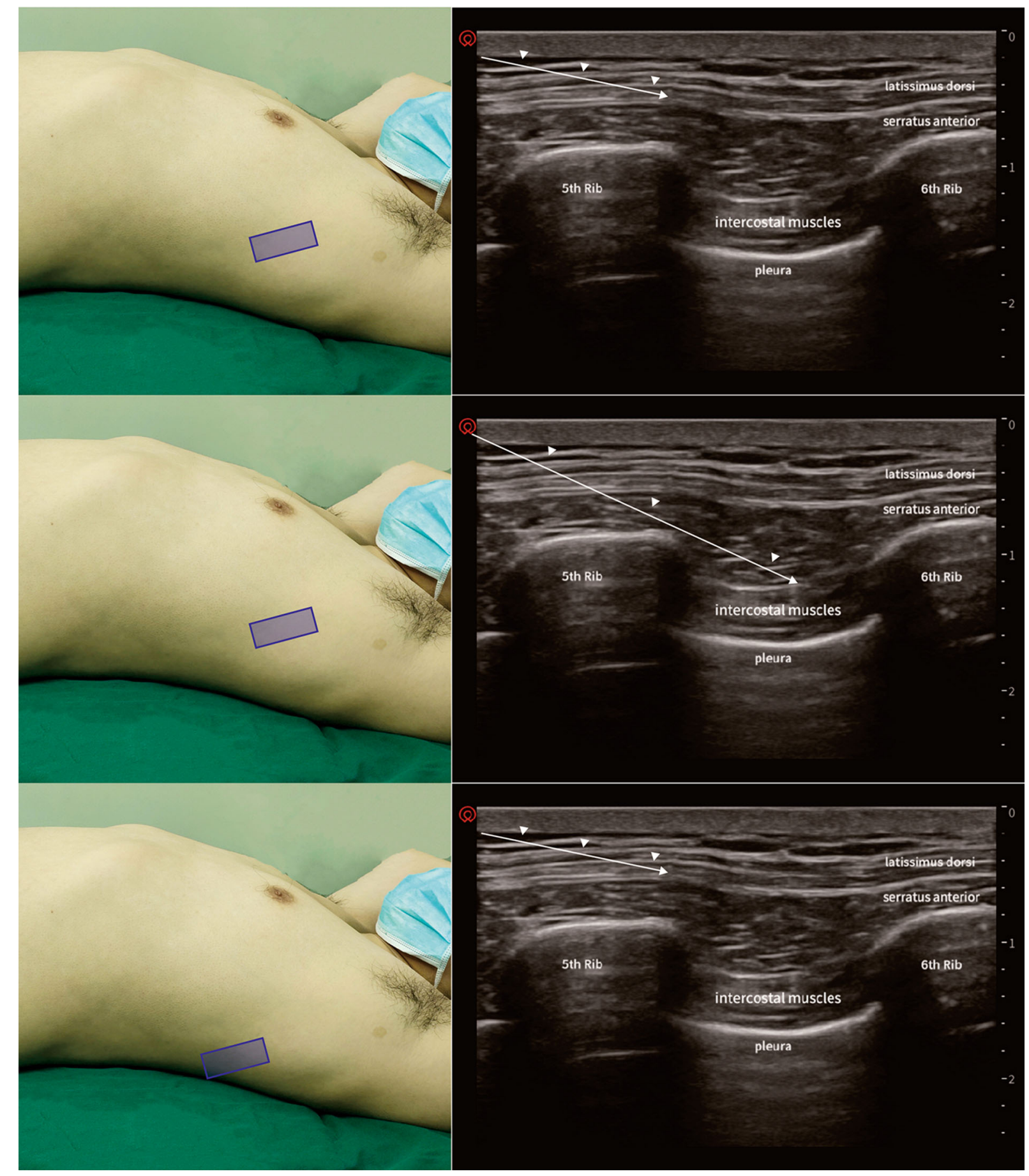

Fig. 2 Graphic representing probe position and ultrasound image obtained during a superficial SAPB (top), seep SAPB (middle), and modified SAPB (bottom)

the range of sensory blockage was tested at T2-T9 in $30 \mathrm{~min}$.

\section{Deep SAPB}

In 2015, Pérez et al. [23] proposed the deep serratus plane block, whereby the ultrasound 
probe was placed under the outer third of the clavicle while the patient was in the supine position. The probe was then slowly moved to the fifth rib in the mid-axillary line. The serratus anterior muscle and intercostal muscle were clearly recognized using ultrasound. A needle was inserted between the serratus anterior muscle and intercostal muscle, and LAs were injected. In summary, the superficial approach is more extensive and longer than the deep block, but the deep serratus anterior block can relieve pain that cannot be controlled by the superficial method.

\section{Modified SAPB}

In 2016, Khemka et al. [24] proposed placing the ultrasound probe obliquely on the second rib and moving the probe downward laterally to the sixth rib in the posterior axillary line. The latissimus dorsi and serratus anterior muscles could be identified under ultrasound, and then $20 \mathrm{ml}$ of $0.25 \%$ levobupivacaine was injected. This method is suitable for patients who undergo breast reconstruction with a latissimus dorsi muscle flap. Although both superficial and deep SAPB can block the long thoracic nerve, which innervates the serratus anterior muscle, these two methods cannot achieve a block of the thoracodorsal nerve. However, the benefit of modified SAPB is the blocking of the thoracodorsal nerve and plexus located under the pectoralis muscle.
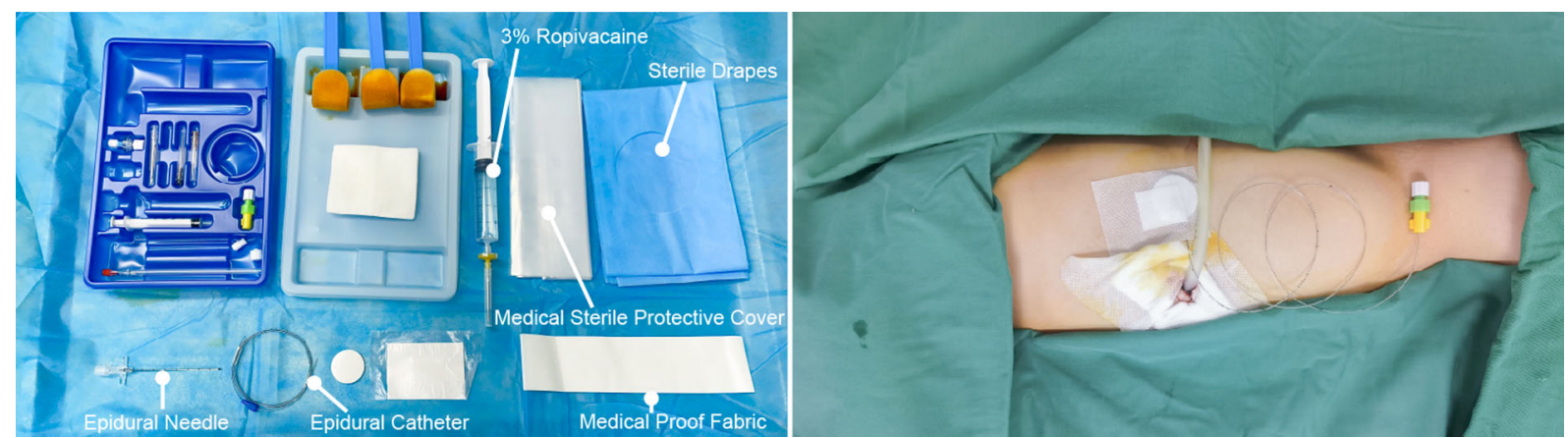

Fig. 3 Devices (left) and anatomical location (right) of continuous SAPB. Cited from: Gao et al. [31]

\section{Continuous SAPB}

Continuous techniques are highly recommended for prolonged analgesia [25, 26], and the continuous SAPB is an effective method of relieving acute pain after a VATS, thoracotomy, and multiple rib fractures [27-30]. In our previous study [31], we successfully implemented a continuous serratus anterior block in patients, and the specific operational details were as follows.

The patient was kept in the surgical position (lateral position), and ultrasound-guided continuous SAPB right after surgery was performed. The advantage of the lateral position was that the operator could sterilize a larger area of the skin. The "scope of disinfection" was very important for catheterization in continuous analgesia. In supine position, the range of disinfection can only reach to the posterior axillary line at most (the distance of the puncture site to the edge of disinfection area may be $<$ $15 \mathrm{~cm}$ ). Therefore, we recommend the lateral position for performing continuous SAPB. After disinfection, the needle was inserted into the fourth or sixth rib, between the anterior axillary line and posterior axillary line, where the latissimus dorsi and the serratus anterior muscle were clearly discernable under ultrasound. Furthermore, $3 \mathrm{ml}$ saline was injected to open the potential interfacial space between the rib and serratus anterior muscle. Afterward, an epidural catheter was passed through. Then, an epidural catheter was threaded through the needle, and we then slowly removed the needle. After a confirmed negative aspiration, $40 \mathrm{ml}$ of $0.375 \%$ 


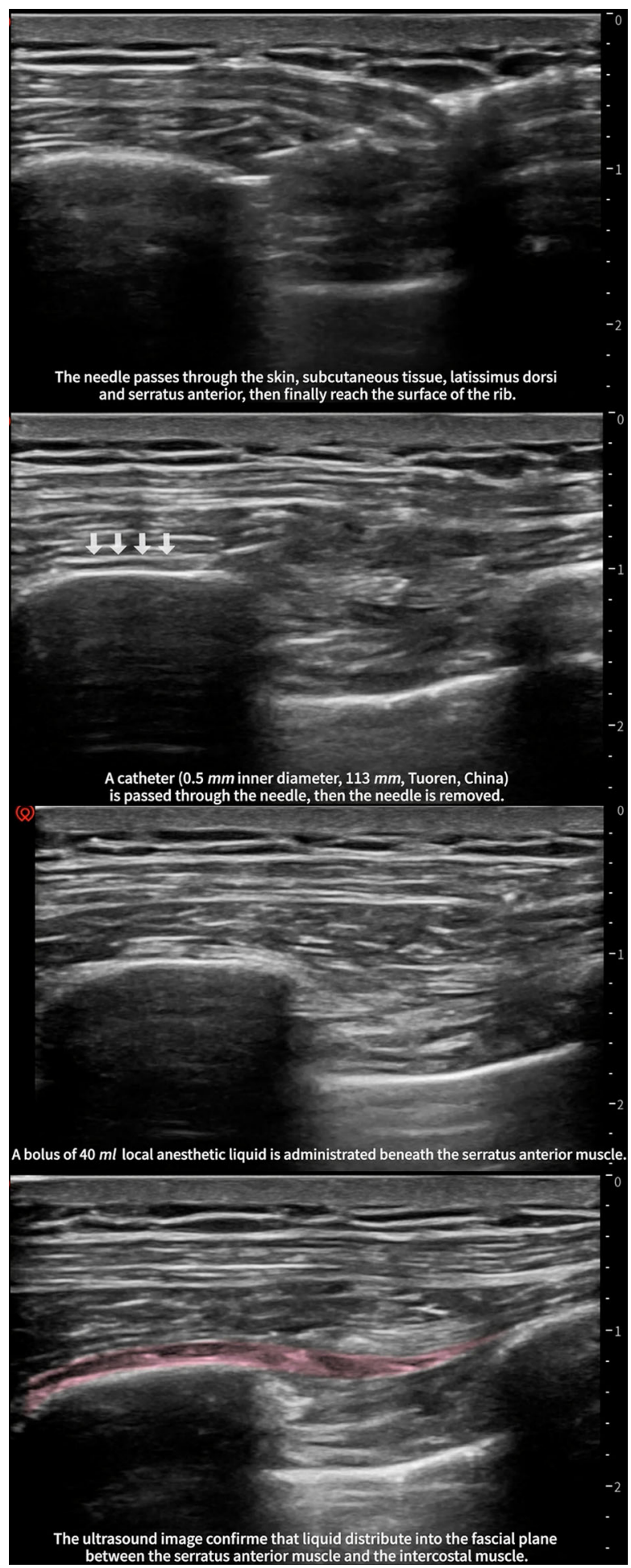

Fig. 4 Procedure of ultrasound-guided continuous serratus anterior plane block ropivacaine was administered beneath the serratus anterior muscle (Fig. 3). The patient-controlled analgesia pump was connected to the catheter, with a background of $0.3 \%$ ropivacaine at a rate of $7 \mathrm{ml} / \mathrm{h}$ (Fig. 4).

\section{Drugs}

According to current research, the commonly used LAs for SAPB are lidocaine, ropivacaine, and bupivacaine. Ropivacaine and bupivacaine have a high safety margin and are the most widely used. The SAPB can be used to maintain a certain duration of analgesia by a single injection or perioperative analgesia by an inplane catheter. In a randomized controlled trial by Huang et al. [32], the analgesic duration and effects of different concentrations of ropivacaine $(0.375 \%, 0.5 \%$, and $0.75 \%)$ were compared in breast surgery. The results showed that the higher the concentration of ropivacaine was, the longer the duration of the sensory blockade. There was no significant difference between 0.5 and $0.75 \%$ ropivacaine compared to postoperative analgesia, but both were superior to the $0.375 \%$ ropivacaine group. Therefore, $0.5 \%$ ropivacaine is more suitable for postoperative analgesia after breast surgery. Furthermore, Kunigo et al. [33] studied the effect of the SAPB drug volume on the anesthetic effect in patients after breast cancer surgery. Although the anesthetic effect of $40 \mathrm{ml}$ of $0.375 \%$ ropivacaine was better than that of $20 \mathrm{ml}$ of $0.375 \%$ ropivacaine, the latter could achieve an optimal effect compared with the former, and it may be safer to avoid the toxic reactions of LAs. Moreover, Kunhabdulla et al. [20] performed the SAPB with $20 \mathrm{ml} 0.125 \%$ levobupivacaine in patients with rib fractures, and it provided safe and effective analgesia to the patients. Currently, there are few clinical studies on the volume and optimal concentration of LAs in SAPB. In the future, more studies are needed to determine the optimal dosing regimen to achieve the desired analgesic effect while avoiding the potential toxic and side effects. 


\section{Adjuvants}

It is risky to prolong the duration of the nerve block by increasing the concentration and dosage of LAs. Therefore, it is necessary to choose other methods to reduce the tissue absorption of LAs, prolong their duration of action, and improve the analgesic effect of regional anesthesia techniques; the addition of adjuvants within LAs is a common method. Some examples of adjuvant medications for regional techniques include dexamethasone, buprenorphine, alpha 2 agonists (such as clonidine and dexmedetomidine), $\mathrm{N}$-methyl-Daspartate antagonists, and non-steroidal antiinflammatory drugs.

Dexmedetomidine, a commonly used adjuvant for LAs, has been shown to have both central and peripheral mechanisms [34, 35]. Abdallah et al. [36] conducted a prospective randomized controlled study in which they administered the continuous SAPB to patients undergoing thoracotomy surgery and randomized them into two groups, one treated with levobupivacaine alone and the other treated with levobupivacaine combined with dexmedetomidine. The results showed that the combined dexmedetomidine group had better analgesic and sedative effects, and levobupivacaine combined with dexmedetomidine significantly reduced the visual analog scale (VAS) scores, occurrence of PPCs, and amount of opioids. To assess the analgesia effect of paravertebral administration of dexmedetomidine as an adjuvant to LAs, Dutta et al. [37] included 30 patients who underwent elective thoracotomy and were assigned randomly to either the ropivacaine (15 $\mathrm{ml} \quad 0.75 \%$ ropivacaine) or dexmedetomidine $(15 \mathrm{ml} \quad 0.75 \%$ ropivacaine plus $0.2 \mu \mathrm{g} / \mathrm{kg}$ of dexmedetomidine) group. They reported that the VAS scores were 5 (2-8) in the Ropin versus 2 (1-6) in Dexem group $2 \mathrm{~h}$ after surgery.

Dexamethasone is a long-acting glucocorticoid that is often used as an adjuvant to LAs. It has been shown that the addition of 4-10 mg of dexamethasone to ropivacaine can prolong the action time, but the existence of a dose-dependent prolongation effect remains uncertain [38]. Moreover, dexamethasone has both local and systemic mechanisms to prolong the analgesic duration of LAs [39]. The local mechanism may stem from the fact that dexamethasone can delay the absorption of LAs by constricting the blood vessels and reducing capillary permeability [40]; the systemic mechanism is likely due to the fact that dexamethasone can inhibit the local cyclooxygenase activity in trauma and reduce the synthesis of pain-causing substances, such as prostaglandins, thus exerting analgesic effects [41]. Mao et al. [42] compared the efficacy of perineural dexamethasone with ropivacaine for TPVB in patients undergoing elective thoracotomy. The VAS scores were $0.55(0.83)$ in the $\mathrm{R}$ group ( $0.5 \%$ ropivacaine) versus 0.24 (0.44) in the RD group (5 $\mathrm{mg}$ dexamethasone and $0.5 \%$ ropivacaine) $6 \mathrm{~h}$ postoperatively.

\section{Block Range}

Several studies have suggested that SAPB may provide postoperative analgesia in certain chest wall procedures without validating the range of blockage of the SAPB. Blanco et al. [17] performed the SAPB bilaterally in four volunteers, and they mapped the specific skin block area with the block site at the fifth intercostal space in the mid-axillary line. Despite the existence of individual differences, the SAPB had a common skin area block, which ranged from $\mathrm{T} 2$ to $\mathrm{T} 6$ for the anterior chest wall, T2 to T8 for the lateral chest wall, and T2 to T9 for the posterior chest wall. In addition, Daga et al. [22] examined the extent of drug diffusion by ultrasound after the bilateral SAPB in seven cadavers; $20 \mathrm{ml}$ water was injected into the serratus anterior plane at the level of the fourth to fifth rib in the posterior axillary line, and the diffusion to the cephalic side was between the second and fourth intercostals and to the caudal side up to the subcostal margin, which is consistent with the results of Blanco. VATS surgical incisions were just located on the block area of SAPB, which ranged from $\mathrm{T} 2$ to $\mathrm{T} 6$ on the anterior chest wall and T2 to T8 on the lateral chest wall. Therefore, the SAPB can achieve effective analgesia in the antero-lateral chest wall. Although there are many recognized regional block 


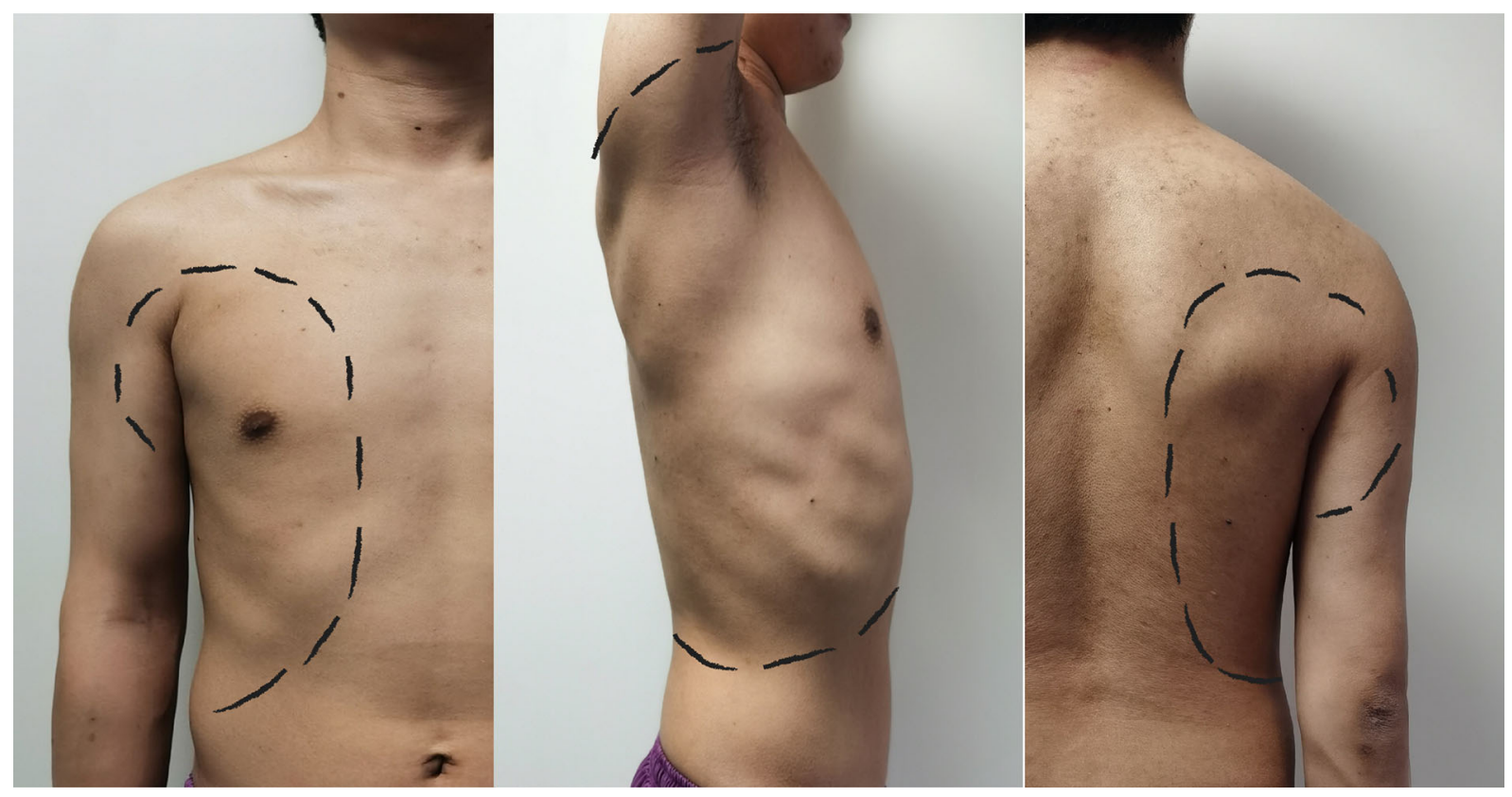

Fig. 5 Block area of SAPB in the anterior chest wall (left), lateral chest wall (middle), and posterior chest wall (right)

methods after VATS, the SAPB plays an irreplaceable role among these methods (Fig. 5).

\section{POSTOPERATIVE PAIN MANAGEMENT}

Although opioid analgesics are commonly applied in postoperative analgesia for VATS, opioids have several limitations owing to opioid-induced adverse effects, such as nausea/ vomiting and respiratory depression [43]. In addition to opioids, there are numerous techniques for regional nerve blocks in the chest wall, including TEA and TPVB, which have been extensively reported in VATS postoperative analgesia [11]. The TEA involves placing a small catheter into the epidural space for neuraxial analgesia. In addition, TEA still carries several risks and limitations, which include hemodynamic instability, intrathecal injection, epidural hematoma, nerve injury, and urine retention. The TPVB technique has some similar complications to TEA; its own list of complications includes pleural puncture (1.1\%), pneumotho$\operatorname{rax}(0.5 \%)$, and vascular puncture (3.8\%) [44]. Both the TEA and TPVB techniques should strictly follow the American Society of Regional
Anesthesia guidelines regarding anticoagulation so that catastrophic complications can be avoided [45]. Experience with TEA and TPVB influences the success of these blocks and is largely institution and physician dependent. Accordingly, this article also discusses the efficacy of SAPB in the management of acute post-surgical pain, with a focus on comparison with those methods.

\section{TEA vs. SAPB}

For a long time, the use of TEA combined with opioids has been considered the gold standard for analgesia after thoracic surgery, and it is also an effective way to reduce pulmonary complications after thoracotomy [46]. However, the TEA technique is demanding and difficult for the operator, requiring not only the placement and removal of catheters but also continuous intraoperative infusion during surgery [47]. It also has a high rate of complications, including accidental dural penetration, a high block level, LA intoxication, and total spinal anesthesia. Besides, postoperative hypotension, neuraxial hematoma, nausea and vomiting, and urinary retention have been frequently reported. 
Furthermore, the use of TEA is limited by a number of contraindications, including local infection at the puncture site, history of spinal surgery, abnormal coagulation function, and oral anticoagulation or antiplatelet drugs. For example, Rajashree et al. [48] first applied the SAPB to thoracic surgery patients with abnormal coagulation and concluded that SAPB can effectively relieve postoperative pain and is safe and easy to implement. Asmaa et al. [49] observed patients undergoing thoracic surgery and found that patients with continuous SAPB were more hemodynamically stable than patients with a continuous epidural block. They also found that the continuous SAPB had an advantage in reducing postoperative opioid use. In addition, the SAPB, a key component of multimodal analgesia after thoracic surgery, is simple to perform and has fewer complications than the other commonly used regional anesthesia techniques.

\section{TPVB vs. SAPB}

The spinal nerve runs in the paravertebral space after piercing out of the intervertebral foramen, where it is not enveloped by fascia. Injection of LAs into this space has good anesthetic and analgesic effects [50]. TPVB is a unilateral block technique; therefore, contralateral respiratory and sympathetic functions can be preserved. This function may be associated with reduced PPCs, postoperative bleeding, and urinary retention [51]. The complications of TPVB are similar to those of TEA; however, spinal cordrelated complications are more likely to occur after TEA, whereas pulmonary complications are more common after TPVB [52]. Hanley et al. [53] compared the analgesic effects of general anesthesia compounded with TPVB and general anesthesia compounded with SAPB in 40 patients undergoing VATS and observed no difference in hemodynamics, opioid side effects, length of hospital stay, or patient satisfaction between the two groups. Further study showed that injection of $20 \mathrm{ml} 0.25 \%$ bupivacaine in the serratus anterior plane to block the sensory afferent nerve (T4-T6) at the thoracoscopic incision site and the intercostal nerve
(T7-T8) at the drain site could effectively relieve the pain associated with the chest tube [54]. Often, TPVB requires several injections to obtain sufficient dermatomes, most commonly at the T3, T5, and T7 levels [55].

\section{Wound Infiltration vs. SAPB}

Wound infiltration includes systematic and extensive surgical site local anesthetic infiltration in the various tissue planes under direct visualization before closure of the surgical incision [56]. LA administration into a surgical wound can block the stimuli that caused by surgical injury at the site of origin. The technique is simple and inexpensive and has a good safety with few side effects. Therefore, it is used widely in many hospitals at the end of surgery. Chen et al. [57] compared the effects of SAPB for postoperative analgesia after thoracoscopic surgery with LA infiltration. Forty adult patients undergoing VATS were randomized to receive either SAPB (SAPB group) or LA infiltration of the incision (LA group). Finally, the SAPB group had lower VAS scores at the 2nd and 8th hours after surgery. Besides, postoperative sufentanil consumption in the SAPB group during $0-8 \mathrm{~h}$ was significantly lower compared with the LA group. Concerns with surgical site infiltration include the potential for local anesthetic systemic toxicity [58]. The absorption of LAs after wound infiltration depends on the dose of LA and the vascularity of the incision site [59]. In fact, several studies have reported that even with larger doses of ropivacaine, which may be higher than recommended (i.e., $400 \mathrm{mg}$ ), the maximum concentration was still much lower than the toxicity threshold [60]. In addition, the use of epinephrine may slow the systemic absorption of LAs. Overall, wound infiltration of LAs is a safe yet under-investigated method of postoperative pain management.

\section{Patient-Controlled Intravenous Analgesia (PCIA) vs. SAPB}

PCIA is a combination of opioid-based and nonopioid drugs, such as nonsteroidal anti-inflammatory drugs (NSAIDs). Although opioids are 
commonly used for postoperative analgesia, they can cause dose-dependent respiratory depression, nausea, vomiting, intestinal obstruction, and other adverse effects [61]. Moreover, nephrotoxicity and gastrointestinal side effects caused by NSAIDs also reduce patients' comfort and satisfaction with anesthesia. The SAPB was first used for postoperative analgesia in breast surgery and has been widely used ever since. Numerous studies have focused on multimodal analgesia combined with regional anesthesia to reduce postoperative pain scores, but they have focused less on reducing opioid consumption and decreasing the incidence of nausea and vomiting [62]. Alessandro et al. [63] conducted a systematic evaluation of patients undergoing SAPB for VATS and included seven studies with 489 patients; the results showed that SAPB had significant advantages in shortening the hospital stay, reducing the consumption of opioids in $24 \mathrm{~h}$ after surgery, and decreasing the incidence of postoperative nausea and vomiting (PONV) in patients undergoing VATS.

A meta-analysis by Alessandro et al. [63] found that the effect of SAPB in reducing perioperative pain was more significant within $6 \mathrm{~h}$ after surgery. Furthermore, Semyonov et al. [16] reported a similar finding; compared to the PCIA group, the SAPB group had a significant advantage only up to $8 \mathrm{~h}$ postoperatively, and there was no significant difference in pain scores after the postoperative 9th hour.

The inability of a single nerve block to provide patients with lasting postoperative analgesia may be related to the single administration pattern and the pharmacokinetic characteristics of the LAs [64]. Intermittent or continuous administration of LAs by placing a catheter in the SAP layer combined with a removable infusion pump can prolong the duration of the SAPB and also facilitate the patient's mobility and recovery, making it a novel regional anesthesia technique. Hanley et al. [53] performed the ultrasound-guided SAPB with catheter placement after VATS, and they found that the continuous SAPB was not inferior to TPVB in terms of $48 \mathrm{~h}$ opioid consumption, and the continuous SAPB was associated with improved functional measures in thoracic surgical patients. Khalil et al. [65] compared the continuous SAPB with TEA for postoperative analgesia after VATS; they found that the continuous SAPB group was not inferior to the TEA group in providing postoperative analgesia, and the difference in VAS at $24 \mathrm{~h}$ postoperatively was not statistically significant. Also, there was no statistically significant difference in the 24-h morphine dosage and the incidence of nausea and vomiting; however, the SAPB group had significantly better circulatory stability than the TEA group. In our previous study, we found that continuous SAPB significantly reduced resting pain compared with traditional PCIA after VATS [66].

\section{SAPB AND LUNG FUNCTION RECOVERY}

Postoperative respiratory impairment may be caused by various factors, including surgical factors (site of incision, postoperative pain, and type of surgical procedure), anesthetic factors (residual anesthetics, injurious mechanical ventilation, and type of anesthesia), and many other patient factors (aging and smoking history) [67]. The operative type has been considered as an important factor that affects postsurgical pulmonary function, particularly when the extent of parenchymal reduction is significant, such as after segmentectomy or lobectomy in VATS [68]. The significant decline in the remaining pulmonary function easily results in a series of PPCs, which refers to a series of respiratory complications after surgery, including lung infection, respiratory failure, pleural effusion, pneumothorax, pulmonary edema, and acute adult respiratory distress syndrome (ARDS) [69]. It is worth noting that pulmonary function exercise is often limited by uncontrolled pain postoperatively. However, postsurgical pain is a modifiable risk factor that can be controlled [70]. Therefore, pain management is valuable for improving lung function recovery and PPCs [71].

Perioperative multimodal analgesia can reduce surgical stress and improve pulmonary function [72, 73]. Matyal et al. [74] conducted a study to compare the immediate postoperative 
pulmonary function of patients between the TPVB group and intercostal nerve block (INB) group after VATS. The results showed that the scores of resting pain and cough pain in both groups were significantly reduced within $4 \mathrm{~h}$ after the operation. On comparing forced expiratory volume in 1-s forced vital capacity and forced expiratory flow $25-75 \%$ between the two groups, it was found that the pulmonary function of patients in the TPVB group was significantly improved compared with that in the INB group. Therefore, it was speculated that adequate analgesia can potentially prevent respiratory complications. A series of studies on SAPB by our group found that in patients undergoing VATS, the pulmonary function in the continuous SAPB group was significantly improved $72 \mathrm{~h}$ after surgery compared with that in the PCIA group, and the incidence of complications, such as pneumonia, atelectasis, hypoxemia, and vomiting, was significantly reduced [31].

\section{CONCLUSIONS}

Postoperative acute pain management and recovery of pulmonary function are important contents of perioperative management of VATS. Effective control of acute postoperative pain can make it possible for patients to perform exercises to improve pulmonary function, and it is one important means of promoting the recovery of pulmonary function. The regional anesthesia technique plays an irreplaceable role in perioperative pain management. The SAPB, an important part of multimodal analgesia after thoracic surgery, has advantages, such as simple operation, safety, and a precise analgesic effect with few complications. The block range of the SAPB covers most of the VATS incisions and location of the chest tube, which can provide better postoperative analgesia for VATS patients. In addition, it can also help in the recovery of lung function, reduce the use of opioids after VATS and the incidence of PONV, and prevent PPCs; hence, it is worthy of clinical promotion. Despite all this, we still need to develop a reasonable scheme and individualize the protocol according to different situations.
However, the SAPB provides us with an important option.

\section{ACKNOWLEDGEMENTS}

Funding. The Key Research and Development Project Foundation of Anhui Province (no. 1804h08020286) provided funding for the study. The Rapid Service Fee was funded by the authors.

Authorship. All named authors meet the International Committee of Medical Journal Editors (ICMJE) criteria for authorship for this article, take responsibility for the integrity of the work as a whole, and have given their approval for this version to be published.

Author Contributions. Jia-qi Chen carried out most of the selection of the articles and drafted the initial manuscript. Xin-lu Yang helped with literature search and collection and compiled pictures and videos, etc. Jia-qi Chen and Xin-lu Yang contributed equally to the article. All the authors contributed to review and critical revision to improve the quality of the paper. All authors have reviewed and approved the final draft of the submitted manuscript.

Disclosures. Jia-qi Chen, Xin-lu Yang, Hai $\mathrm{Gu}$, Xiao-qing Chai, and Di Wang have nothing to disclose.

Compliance with Ethics Guidelines. This article is based on previously conducted studies and does not contain any new studies with human participants or animals performed by any of the authors.

Open Access. This article is licensed under a Creative Commons Attribution-NonCommercial 4.0 International License, which permits any non-commercial use, sharing, adaptation, distribution and reproduction in any medium or format, as long as you give appropriate credit to the original author(s) and the source, provide a link to the Creative Commons licence, and 
indicate if changes were made. The images or other third party material in this article are included in the article's Creative Commons licence, unless indicated otherwise in a credit line to the material. If material is not included in the article's Creative Commons licence and your intended use is not permitted by statutory regulation or exceeds the permitted use, you will need to obtain permission directly from the copyright holder. To view a copy of this licence, visit http://creativecommons.org/licenses/by$\mathrm{nc} / 4.0 /$.

\section{REFERENCES}

1. Petersen RH, Holbek BL, Hansen HJ, Kehlet $H$. Video-assisted thoracoscopic surgery-taking a step into the future. Eur J Cardiothorac Surg. 2017;51(4): 694-5.

2. Umari M, Carpanese V, Moro V, et al. Postoperative analgesia after pulmonary resection with a focus on video-assisted thoracoscopic surgery. Eur J Cardiothorac Surg. 2018;53(5):932-8.

3. Bai Y, Sun K, Xing X, et al. Postoperative analgesic effect of hydromorphone in patients undergoing single-port video-assisted thoracoscopic surgery: a randomized controlled trial. J Pain Res. 2019;12: 1091-101.

4. Zhang X, Zhang C, Zhou X, et al. Analgesic effectiveness of perioperative ultrasound-guided serratus anterior plane block combined with general anesthesia in patients undergoing video-assisted thoracoscopic surgery: a systematic review and metaanalysis. Pain Med. 2020;21(10):2412-22.

5. Jiang $\mathrm{C}, \mathrm{Li} \mathrm{Y}, \mathrm{Li} \mathrm{X}$, et al. Postoperative analgesic effect of acupotomy combined with patient-controlled analgesia in patients undergoing video-assisted thoracoscopic surgery: a study protocol for a randomized controlled trial. Trials. 2020;21(1):998.

6. Steinthorsdottir KJ, Wildgaard L, Hansen HJ, Petersen RH, Wildgaard K. Regional analgesia for video-assisted thoracic surgery: a systematic review. Eur J Cardiothorac Surg. 2014;45(6):959-66.

7. Colley CM, Fleck A, Goode AW, Muller BR, Myers MA. Early time course of the acute phase protein response in man. J Clin Pathol. 1983;36(2):203-7.

8. Wallace AM, Sattar N, Mcmillan DC. The co-ordinated cytokine/hormone response to acute injury incorporates leptin. Cytokine. 2000;12(7):1042-5.
9. Erb J, Orr E, Mercer CD, Gilron I. Interactions between pulmonary performance and movementevoked pain in the immediate postsurgical period: implications for perioperative research and treatment. Reg Anesth Pain Med. 2008;33(4):312-9.

10. Batchelor TJP, Rasburn NJ, Abdelnour-Berchtold E, et al. Guidelines for enhanced recovery after lung surgery: recommendations of the Enhanced Recovery After Surgery $\left(\right.$ ERAS $\left.^{\circledR}\right)$ Society and the European Society of Thoracic Surgeons (ESTS). Eur J Cardiothorac Surg. 2019;55(1):91-115.

11. Campos JH, Peacher D. Choosing the best method for postoperative regional analgesia after video-assisted thoracoscopic surgery. J Cardiothorac Vasc Anesth. 2020;34(7):1877-80.

12. Mathew B, Lennon FE, Siegler J, Mirzapoiazova T, Mambetsariev N, Sammani S, et al. The novel role of the mu opioid receptor in lung cancer progression: a laboratory investigation. Anesth Analg. 2011;112:558-67.

13. Steegers MA, Snik DM, Verhagen AF, van der Drift $\mathrm{MA}$, Wilder-Smith $\mathrm{OH}$. Only half of the chronic pain after thoracic surgery shows a neuropathic component. J Pain. 2008;9(10):955-61.

14. Piccioni F, Segat M, Falini S, et al. Enhanced recovery pathways in thoracic surgery from Italian VATS Group: perioperative analgesia protocols. J Thorac Dis. 2018;10(Suppl 4):S555-63.

15. Blanco R, Parras T, McDonnell JG, et al. Serratus plane block: a novel ultrasound-guided thoracic wall nerve block. Anaesthesia. 2013;68:1107-13.

16. Semyonov M, Fedorina E, Grinshpun J, et al. Ultrasound-guided serratus anterior plane block for analgesia after thoracic surgery. J Pain Res. 2019;12: 953-60.

17. Lung K, St Lucia K, Lui F. Anatomy, thorax, serratus anterior muscles. Treasure Island: StatPearls Publishing; 2020.

18. Durant E, Dixon B, Luftig J, et al. Ultrasound-guided serratus plane block for ED rib fracture pain control. Am J Emerg Med. 2017;35(1):197.e3-197. e6.

19. Zocca JA, Chen GH, Puttanniah VG, et al. Ultrasound guided serratus plane block for treatment of postmastectomy pain syndromes in breast cancer patients: a case series. Pain Pract. 2017;17(1): 141146.

20. Kunhabdulla NP, Agarwal A, Gaur A, et al. Serratus anterior plane block for multiple rib fractures. Pain Physician. 2014;17(4):E553-5. 
21. Mayes J, Davison E, Panahi P, et al. An anatomical evaluation of the serratus anterior plane block. Anaesthesia. 2016;71(9):1064-9.

22. Daga V, Narayanan MK, Dedhia JD, et al. Cadaveric feasibility study on the use of ultrasound contrast to assess spread of injectate in the serratus anterior muscle plane. Saudi J Anaesthesia. 2016;10(2):198.

23. Pérez Herrero MA, López lvarez S, Fadrique Fuentes A, et al. Quality of postoperative recovery after breast surgery. General anaesthesia combined with paravertebral versus serratus-intercostal block. Rev Esp Anestesiol Reanim. 2016;63(10):564-71.

24. Khemka R, Chakraborty A, Ahmed R, et al. Ultrasound-guided serratus anterior plane block in breast reconstruction surgery. A A Case Rep. 2016;6(9): 280-2.

25. Chou R, Gordon DB, de Leon-Casasola OA, et al. Management of postoperative pain: a clinical practice guideline from the American Pain Society, the American Society of Regional Anesthesia and Pain Medicine, and the American Society of Anesthesiologists' Committee on Regional Anesthesia, Executive Committee, and Administrative Council. J Pain. 2016;17:131-57.

26. Vorobeichik L, Brull R, Bowry R, et al. Should continuous rather than single-injection interscalene block be routinely offered for major shoulder surgery? A meta-analysis of the analgesic and side-effects profiles. Br J Anaesth. 2018;120:679-92.

27. Madabushi R, Tewari S, Gautam SK, et al. Serratus anterior plane block: a new analgesic technique for post-thoracotomy pain. Pain Physician. 2015;18: E421-424.

28. Reyad RM, Shaker EH, Ghobrial HZ, et al. The impact of ultrasoundguided continuous serratus anterior plane block versus intravenous patientcontrolled analgesia on the incidence and severity of postthoracotomy pain syndrome: a randomized, controlled study. Eur J Pain. 2020;24:159-70.

29. Kunhabdulla NP, Agarwal A, Gaur A, et al. Serratus anterior plane block for multiple rib fractures. Pain Physician. 2014;17:553-5.

30. Fu P, Weyker PD, Webb CA. Case report of serratus plane catheter for pain management in a patient with multiple rib fractures and an inferior scapular fracture. A A Case Rep. 2017;8:132-5.

31. Gao W, Yang XL, Hu JC, et al. Continuous serratus anterior plane block improved early pulmonary function after lung cancer surgery. Ann Thorac Surg. 2021. https://doi.org/10.1016/j.athoracsur. 2021.02.032.
32. Huang L, Zheng L, Wu B, et al. Effects of ropivacaine concentration on analgesia after ultrasoundguided serratus anterior plane block: a randomized doubleblind trial. J Pain Res. 2020;13:57-64.

33. Kunigo T, Murouchi T, Yamamoto S, et al. Injection volume and anesthetic effect in serratus plane block. Reg Anesth Pain Med. 2017;42:737-40.

34. Nguyen V, Tiemann D, Park E, et al. Alpha-2 agonists. Anesthesiol Clin. 2017;35(2):233-45.

35. Tang C, Xia Z. Dexmedetomidine in perioperative acute pain management: a non-opioid adjuvant analgesic. J Pain Res. 2017;10:1899-904.

36. Abdallah NM, Bakeer AH, Youssef RB, et al. Ultrasoundguided continuous serratus anterior plane block: dexmedetomidine as an adjunctive analgesic with levobupivacaine for post-thoracotomy pain. A prospective randomized controlled study. J Pain Res. 2019;12:1425-31.

37. Dutta V, Kumar B, Jayant A, Mishra AK. Effect of continuous paravertebral dexmedetomidine administration on intraoperative anesthetic drug requirement and post-thoracotomy pain syndrome after thoracotomy: a randomized controlled trial. J Cardiothorac Vasc Anesth. 2017;31(1):159-65.

38. Albrecht E, Reynvoet M, Fournier N, et al. Doseresponse relationship of perineural dexamethasone for interscalene brachial plexus block: a randomised, controlled, triple-blind trial. Anaesthesia. 2019;74(8):1001-8.

39. Marhofer P, Columb M, Hopkins PM, et al. Dexamethasone as an adjuvant for peripheral nerve blockade: a randomised, triple-blinded crossover study in volunteers. Br J Anaesth. 2019;122(4): 525-31.

40. Chong MA, Szoke DJ, Berbenetz NM, et al. Dexamethasone as an adjuvant for caudal blockade in pediatric surgical patients: a systematic review and meta-analysis. Anesth Analg. 2018;127(2):520-8.

41. Zhu C, Zhang S, Gu Z, et al. Caudal and intravenous dexamethasone as an adjuvant to pediatric caudal block: a systematic review and metaanalysis. Paediatr Anaesth. 2018;28(3):195-203.

42. Mao Y, Zuo Y, Mei B, et al. Efficacy of perineural dexamethasone with ropivacaine in thoracic paravertebral block for postoperative analgesia in elective thoracotomy: a randomized, double-blind, placebo-controlled trial. J Pain Res. 2018;11: 1811-9.

43. Nobel TB, Adusumilli PS, Molena D. Opioid use and abuse following video-assisted thoracic surgery 
(VATS) or thoracotomy lung cancer surgery. Transl Lung Cancer Res. 2019;8(Suppl 4):S373-7.

44. Lönnqvist PA, MacKenzie J, Soni AK, Conacher ID. Paravertebral blockade. Failure rate and complications. Anaesthesia. 1995;50(9):813-5.

45. Horlocker TT, Vandermeuelen E, Kopp SL, et al. Regional anesthesia in the patient receiving antithrombotic or thrombolytic therapy: American Society of Regional Anesthesia and Pain Medicine evidence-based guidelines (fourth edition). Reg Anesth Pain Med. 2018;43(3):263-309.

46. Gottschalk A, Cohen SP, Yang S, et al. Preventing and treating pain after thoracic surgery. Anesthesiology. 2006;104:594-600.

47. Takimoto K, Nishijima K, Ono M. Serratus plane block for persistent pain after partial mastectomy and axillary node dissection. Pain Physician. 2016;19(3):481-6.

48. Madabushi R, Tewari S, Gautam SK, et al. Serratus anterior plane block: a new analgesic technique for post-thoracotomy pain. Pain Physician. 2015;18(3): E421-4.

49. Asmaa EK, Nasr MA, Ghada MB, et al. Ultrasound guided serratus anterior plane block versus thoracic epidural analgesia for thoracotomy pain. J Cardiothorac Vasc Anesth. 2017;31(1):152-8.

50. Kadomastu Y, Mori S, Ueno H, et al. Comparison of the analgesic efforts of modified continuous intercostal block and paravertebral block under surgeon's direct vision after video-assisted thoracic surgery: a randomized clinical trail. Gen Thorac Cardiovasc Surg. 2018;66(7):425-31.

51. Qiu Y, Wu J, Huang Q, et al. Acute pain after serratus anterior plane or thoracic paravertebral blocks for video-assisted thoracoscopic surgery: a randomised trial [published online ahead of print, 2020 May 15] [retracted in: Eur J Anaesthesiol. 2020 Jul;37(7):624]. Eur J Anaesthesiol.

52. Norum HM, Breivik H. A systematic review of comparative studies indicates that paravertebral block is neither superior nor safer than epidural analgesia for pain after thoracotomy. Scand J Pain. 2017;1(1):12-23.

53. Hanley C, Wall T, Bukowska I, et al. Ultrasoundguided continuous deep serratus anterior plane block versus continuous thoracic paravertebral block for perioperative analgesia in videoscopic-assisted thoracic surgery. Eur J Pain. 2020;24(4): 828-38.

54. Chu GM, Jarvis GC. Serratus anterior plane block to address postthoracotomy and chest tube-related pain: a report on 3 cases. A A Case Rep. 2017;8(12): 322-5.

55. Pennefather SH, Quarterman PJ, Klinger RY, et al. Pain management after thoracic surgery. In: Slinger P, editor., et al., Principles and practice of anesthesia for thoracic surgery. New York: Springer; 2011. p. 675-99.

56. Joshi GP, Machi A. Surgical site infiltration: a neuroanatomical approach. Best Pract Res Clin Anaesthesiol. 2019;33(3):317-24.

57. Chen G, Li Y, Zhang Y, Fang X. Effects of serratus anterior plane block for postoperative analgesia after thoracoscopic surgery compared with local anesthetic infiltration: a randomized clinical trial. J Pain Res. 2019;12:2411-7.

58. Neal JM, Bernards CM, Butterworth JF 4th, et al. ASRA practice advisory on local anesthetic systemic toxicity. Reg Anesth Pain Med. 2010;35(2):152-61. https://doi.org/10.1097/AAP.0b013e3181d22fcd.

59. Rawal N. Current issues in postoperative pain management. Eur J Anaesthesiol. 2016;33(3): 160-71.

60. Stringer BW, Singhania AK, Sudhakar JE, Brink RB. Serum and wound drain ropivacaine concentrations after wound infiltration in joint arthroplasty. J Arthroplasty. 2007;22(6):884-92.

61. Palmer PP, Miller RD. Current and developing methods of patient controlled analgesia. Anesthesiol Clin. 2010;28:587-99.

62. Chong M, Berbenetz N, Kumar K, et al. The serratus plane block for postoperative analgesia in breast and thoracic surgery: a systematic review and metaanalysis. Reg Anesth Pain Med. 2019;44:1066-74.

63. De Cassai A, Boscolo A, Zarantonello F, et al. Serratus anterior plane block for video-assisted thoracoscopic surgery: a meta-analysis of randomised controlled trials. Eur J Anaesthesiol. 2021;38(2): 106-14.

64. Meierhenrich R, Hock D, Kühn S, et al. Analgesia and pulmonary function after lung surgery: is a single intercostal nerve block plus patient-controlled intravenous morphine as effective as patient-controlled epidural anaesthesia? A randomized non-inferiority clinical trial. Br J Anaesth. 2011;106(4):580-9.

65. Khalil AE, Abdallah NM, Bashandy GM, et al. Ultrasound guided serratus anterior plane block versus thoracic epidural analgesia for thoracotomy pain. J Cardiothorac Vasc Anesth. 2017;31(1): 152-8. 
66. Yang XL, Gu H, Hu JC, et al. Operation, effectiveness, and limitations of continuous serratus anterior plane blocks for thoracoscopic surgery in adults. J Pain Res. 2020;13:2401-10.

67. Kocher GJ, Gioutsos KP, Ahler M, et al. Perioperative lung function monitoring for anatomic lung resections. Ann Thorac Surg. 2017;104(5):1725-32.

68. Kavanagh BP, Katz J, Sandler AN. Pain control after thoracic surgery. A review of current techniques. Anesthesiology. 1994;81(3):737-59.

69. Meierhenrich $\mathrm{R}$, et al. Analgesia and pulmonary function after lung surgery: is a single intercostal nerve block plus patientcontrolled intravenous morphine as effective as patient-controlled epidural anaesthesia? A randomized non-inferiority clinical trial. Br J Anaesth. 2011;106(4):580-9.

70. Hedenstierna G, Edmark L. Effects of anesthesia on the respiratory system. Best Pract Res Clin Anaesthesiol. 2015;29(3):273-84.
71. Jammer I, Wickboldt N, Sander M, et al. Standards for definitions and use of outcome measures for clinical effectiveness research in perioperative medicine: European Perioperative Clinical Outcome (EPCO) definitions: a statement from the ESAESICM joint taskforce on perioperative outcome measures. Eur J Anaesthesiol. 2015;32(2):88-105.

72. Detterbeck FC. Efficacy of methods of intercostal nerve blockade or pain relief after thoracotomy. Ann Thorac Surg. 2005;80(4):1550-9.

73. Gottschalk A, et al. Preventing and treating pain after thoracic surgery. Anesthesiology. 2006;104(3): 594-600.

74. Matyal R, Montealegre-Gallegos M, Shnider M, et al. Preemptive ultrasound-guided paravertebral block and immediate postoperative lung function. Gen Thorac Cardiovasc Surg. 2015;63(1):43-8. 